\title{
Strategies to Improve Speed Reading and Its Effect on Comprehension in Student Teachers of English- An Experimental Study
}

\author{
F L.Bapitha.S.Gunasekaran
}

\begin{abstract}
Reading is not only one of the most important skills in language learning, but also one of the main objectives of learning English in general. Many factors such as students' background knowledge, motivation, interest, organization of the texts and study skills may affect reading skill. This paper describes an experimental study to identify the effect of the significance of speed reading on comprehension. The results indicate that there are significant gains as a result of the experiment. Reading became faster, scientific and systematic.

Keywords: Speed Reading, Comprehension, Strategies, Experiment, Scientific, Systematic
\end{abstract}

\section{INTRODUCTION}

Man is a social being and has to live in a society conveying his thoughts and ideas to another person using a medium and a language. The language is the medium and the instrument through which ideas are transmitted from one mind to another. Language is a system of communication as such it is essential for human society. Every society has its own language. There are many languages and among those languages English is the common and popular language which people are quite familiar with in all walks of life.

English occupies today the pride of place as a universal language. It is a means of international communication. In Indian schools, English is a compulsory subject of study from first standard to twelfth standard. It is, therefore, essential that its fundamental skills of understanding, speaking, reading and writing are to be emphasized in any teaching-learning situation. Reading occupies a special place in the complicity of language skills, which are listening, speaking and writing. As Reading proficiency is the royal road to knowledge, it is essential to success in all academic subjects. In modern life, learning depends largely upon one's ability to interpret the printed page accurately and fully.

Revised Manuscript Received on October 30, 2019.

* Correspondence Author

F L.Bapitha*, Assistant Professor of English Anna University University College of Engineering

Ramanathapuram, Tamilnadu, India

Dr.S.Gunasekaran Assistant Professor of English Anna University University College of Engineering Dindigul, Tamilnadu, India

(c) The Authors. Published by Blue Eyes Intelligence Engineering and Sciences Publication (BEIESP). This is an open access article under the CC BY-NC-ND license (http://creativecommons.org/licenses/by-nc-nd/4.0/)
Comprehension carries the understanding of a word or a phrase by reading and recognition to the understanding of the meaning intended by the author. Reading without comprehension is not reading at all. Comprehension and reading go hand in hand. Reading with understanding requires a relationship between the conscious and the subconscious. Speed reading means reading the printed line with efficiency, direction, attention and constant awareness. There are many kinds of reading speeds which depend on the difficulty of the material being read as well as the students. The first reading speed might be called 'study speed', this is the slowest reading speed and is used for difficult material.

The second reading speed would include easier text books, novels, and so on. Average reading speed can be used in most prose material whether factual, descriptive or narrative. Skimming and Scanning are the two very important advanced skills of reading. Scanning is also a skill, which demands a quick answer to a specific question. Skimming differs from Scanning in that the reader is not looking for the answer to a specific question, but is only concerned with the over all picture of the reading matter.

There are various factors which affect the reading efficiency of individuals. They are vocalization, sub-vocalization, head movements, supports,word-for-word reading and regression. Identifying the very significance of the effect of speedreading on comprehension, an experimental study has been undertaken. The student teachers were chosen as sample of the experiment due to the role played by them in real classrooms.

\section{RESEARCH QUESTIONS}

The following research questions were formed:

1.What is the effect of an experimental package of reading programme on the student teachers exposed to treatment individually?

2. What is the effect of experimental package of reading programme in 100 student teachers as a group?

3. What is the differential effect of the experimental treatment on student teachers from rural and urban areas?

4. What is the differential effect of the experimental treatment on student teachers of English and Tamil medium?

5. What is the differential effect of the experimental treatment on men and women student teachers?

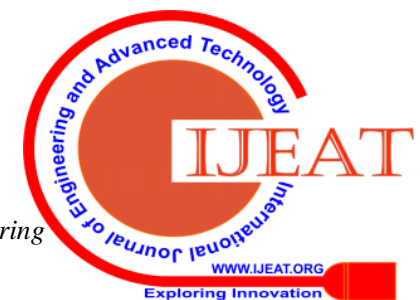




\section{Strategies to Improve Speed Reading and Its Effect on Comprehension in Student Teachers of English-An Experimental Study}

6. What is the reaction of the participant student teachers in a class belonging to various strategies that support the acquisition of reading skills?

7. What is the relationship between speed and comprehension scores?

\section{REVIEW OF RELATED LITERATURE}

The present study on the reading effectiveness of student teachers has an intimate similarity with the studies of Anasurya (2013), Srivastava (2013), Gowan and Schieibal (2014) and Quantz (2013). These studies applied themselves to an experimental verification of the incremental values in the reading abilities of the subjects who were exposed systematically to some form of reading promotion techniques and strategies.

The results are uniformly positive and productive while this situation has been established by many a researcher, there seems to be a limitation on the reading strategy packages used and no attempt was made on the student teachers on whom the implementation programme, squarely, depends in a school situation.

Anasurya (2013) found that the reading efficiency of speed and comprehension were related to students' performance of secondary level. According to Poulton (2012), the average reading normally lies in between 340-350 words-per-minute, but Maria Tera of the Philippines with training in speed reading has acquired the speed of reading 750 words-perminute.

Srivastava (2013) in an experiment with senior executives found that there was a positive significant relationship between reading speed and comprehension. Tinker (2013) found that the correlation between rate of work on reading tests and comprehension was very high for easy materials, but it decreased steadily as the difficulty of the material increased. Husbands and Shores (2013) also found that there was a substantial relationship between rate and comprehension only in case of easy reading materials. Similarly, Gowan and Scheibel (1960) found that an intensive training in speed-reading helped the tenth grade English students to gain the speed of reading from 284 to 896 words -per-minute while gaining in comprehension.

\section{RESEARCH DESIGN}

The present study on the experimental verification of the strategies of speed-reading and comprehension on student teachers took its strength from its close similarities to the studies reported above. It also involves a repeated treatments design of a series of treatments. However, a carefully controlled and administered experiment with a welldesigned treatment package and validated tests of measurements would yield dependable and generalisable results to the maximum extent. Basically, the present study is a popular experimental design with two equivalent groups of student teachers, experimental and controlled, a pre-test and final test with an intermediate test. The treatment package consists of a series of treatment instructions to be implemented one after the other to attain mastery of reading skills and gains from the experiment are measured and analyzed.
The experiment consists of two approaches. A group of 100 student teachers were given a treatment package of instructions on the development of reading with special focus on the aspects, that include reading with speed and comprehension, namely, 1.Vocalization 2.Sub-vocalization 3. Head movements 4. Supports 5. Word-for-word reading, and 6. Regressions.

The experimental group was given adequate time to go through the instructions and in a general discussion, with experienced teacher educators in English methodology their questions were answered, precautions were identified, strategies demonstrated, and they were strongly advised to learn and implement techniques to improve their reading efficiency. A second small group of five student teachers was selected to whom the same experimental treatment package was given and they were subjected to individual guidance, trial and error, correction and feed back in a classroom situation over a period of time.

Experienced teacher educators in English had given individualized instruction and guidance continuously on all aspects of the techniques of reading mentioned earlier.

Therefore, broadly, the study procedure involves

1. Preparation of the instructional package on reading.

2. Administration to five student teachers individually.

3. Administration to a large group of 100 student teachers.

4. Testing the criterion behavior using popular tests from authorized sources, using selected passages and questions that follow the passages.

5. An evaluative questionnaire to student teachers to diagnose their difficulties and to determine priorities for future experimentation.

The experimental study is designed in a straight and direct approach. As the initial conditions were made equivalent, and the experimental treatment was made on systematic lines based on an accepted theoretical framework of exercises of equal difficulty and length and tests that were validated, the results tend to be general sable.

\section{RESULTS}

The student teachers who had no idea of the importance of reading, no awareness of the various physical gestures that hamper the acquisition of speed in reading, and no instructional training exercise, so far, reacted very positively, absorbed the system very sincerely, and pursued the processes very studiously. Hence, the results were very much in favour of the experimental outcomes and yielded significant gains in speed-reading. This awareness and alertness in reading with speed minimizing the impediments had its influencing effect on comprehension of content of material read where the student teachers' abilities improved correspondingly. A primary study of the progress of the achievement of experimental individual small group in comparison with the controlled small group of five individuals is very revealing. The two groups were equivalent to begin with. But the enormous improvement of the experimental individuals clearly demonstrated that the personal attention and intensive training resulted in a much higher quantum of reading achievement and hence comprehension. 
The control group remained static and has shown some slight indications of decrease in the measures. The group experiences were found to be, conspicuously different in favour of small group treatment.

The incremental values between the large and small groups clearly suggest that individual approach and presentation factor by factor, rectifying the defects then and there, giving remedial suggestions, and attending to individual needs and problems on one-to-one basis are more effective than large class room independent study situations.

The small group was much lower in its pre-test status than large group on words-per-minute. However, due to the experimental influence, the small group individual student teachers have been found functioning at a much higher level, than large group at the final-test.

The improvement of the small group from its initial stage to its final stage was in itself a phenomenal and significant even capable of being accepted and generalized.

In comprehension, the two groups started equally and the individual group had greater gains as seen from an average score of eight out of a possible ten. Some have scored all questions correctly. At this juncture, this finding tends to indicate a positive dependency relationship beween speed and comprehension.

Both the correlations are very high and positive. A positive relationship can be seen between words-per-minute and comprehension scores. As the speed-reading is increasing, comprehension scores are also increasing. When reading is done carefully, eliminating all distractions and interferences, Reading becomes not only faster but also meaningful resulting in better understanding and comprehension of the content of the passages read. The experiment was not meant only to increase the speed of reading, but the focus was also on reading faster scientifically and systematically. An element of discipline that ensured attention, concentration of mind, and accuracy was ingrained in the experimental package which, consequently, promoted Reading with Speed with Comprehension. Hence, the relationship between words-per-minute and comprehension scores was found to be significant.

The fact that a student teacher was a man or woman did not matter as far as the achievement scores on words-per-minute tests were concerned. The hypothesis that women would do better and that there would be a significant difference in sex variable was not true in this context. Reading skills are equally new to the two groups of subjects.

Also, noticeable differential activities of criterion measurements were noted on subgroup comparisons in favour of English medium students and urban based students as against Tamil medium students and rural based students respectively.

The gains in speed-reading were conspicuously higher in small group individual attention package than large group instruction. This occurrence strongly supports the classroom teaching dynamics that recommend individualized instruction.

\section{CONCLUSION AND RECOMMENDATIONS}

The study includes training student teachers in the art and craft of speed-reading with better comprehension. The far-

reaching aim was to inculcate the strategies for speedreading, which would have to be translated in to action in the regular school situations of teaching of English. As such, a descriptive survey study may be undertaken to explore the present teaching of reading skills into classrooms by English Language teachers and the extent to which they are taking care of the factors retarding speed. A study may be undertaken to identify the most effective retarding factors in developing Speed Comprehension skill in student teachers and also development of different types of reading programmes for student teachers and testing their efficiency in teacher educators programmes. Also, a longitudinal study may be made on the present student teachers who should be teachers in schools to verify and establish the extent to which they were able to do justice to the gains they had demonstrated in the present experimental research.

This concept of studying the reading skills may be extended to college levels and even to normal adult populations in various vocations. Descriptive and experimental research on a more intensive level can be undertaken on specific factors like Vocalization and Regression which when properly attended to might cause changes in the reading skills of subjects towards desirable goals.

The whole teacher education curriculum should be revamped by making reading an essential and basic skill required of all students at all levels. A certain minimum reading competency should be prescribed at different levels as a circular credit requirement for academic progress. To this extent, in service and pre-service training modules ought to be revised suitably.

Reading is not specific and exclusive to the English Language Teachers. It is the responsibility of every teacher of Mathematics, Sciences or Social Sciences, to deliberately focus their attention on this basic skill, which will enhance and accelerate the understanding and comprehension stages on the cognitive ladder. It is stressed and strongly recommended that educators bestowing attention on the ills and maladies of acquisition of speed-reading with comprehension should propose programmes and activities in the curriculum, which ensure the fundamentally required skills of speed-reading.

\section{REFERENCES}

1. 1.Anasurya (2013). Cited from Reading Efficiency at P.U.C level in English. Unpublished Thesis, Hyderabad: CIEFL

2. Artley, A Sled. The Development of Reading Maturity in High School. Implication of Gray-Rugers Study. Educational Administration and Supervision. 43: 321-28, Oct. 1957

3. Athreya, N.H. (2013). Speed Reading. Delhi: Vision Books Private Limited.

4. Azad, Maulana Abul Kalam. Cited from Bhatia, KK (2013) New Techniques of Teaching English as a Foreign Language. Chandigarh: NBS Educational Publishers.

5. Bayley, Harry (2013) Quicker Reading. New York: Pitman Publishing Corporation.

6. Best, John IX., (2013). Research in Education. Fourth Edition. New Delhi: Prentice-Hall of India Private Limited.

7. Betts, Emmett Albert (2013) Foundations of Reading Instruction. Cincinnati: American Book Company.

8. Bhagya Lakshmi L., Author and Digumarti Bhaskara Rao, Editor (2000); Reading and Comprehension. New Delhi. Discovery Publishing Home. 
Strategies to Improve Speed Reading and Its Effect on Comprehension in Student Teachers of English-An Experimental Study

9. Bhatia, KK., (2013) Measurement and Evaluation in Education. Ludhiana: Prakash Brothers.

10. Bird, C., (1931). Effective Study Habits. New York: Appleton-Century Crofts.

11. Bond and Bond, E., (2012). Development Reading in High School. New York: McMillan Company.

12. Bond, G.L (2012). Teaching the child to Read. New York: The McMillan Company.

13. Paul D.Leedy (2013). Reading Importance of Adults. New York McGraw-Hill Company.

14. Rosch, Reymand, "A Teaching Desirable Study Habits through Experimentation" Catholic Educational Review. 51:53-62, 1953 Spach, G.D., (1963). Towards Better Reading. Gerrad: Champaign, Inc. 